\title{
Atomic-scale properties of Ni-based FCC ternary, and quaternary alloys
}

\author{
Artur Tamm $^{\mathrm{a}, \mathrm{b}, *}$, Alvo Aabloo ${ }^{\mathrm{b}}$, Mattias Klintenberg $^{\mathrm{c}}$, Malcolm Stocks ${ }^{\mathrm{d}}$, \\ Alfredo Caro ${ }^{\mathrm{a}}$ \\ ${ }^{a}$ Materials Science and Technology Division, Los Alamos National Laboratory Los Alamos \\ NM 87544, USA \\ ${ }^{b}$ IMS Lab, Institute of Technology, University of Tartu, 50411 Tartu, Estonia \\ ${ }^{c}$ Department of Physics and Astronomy, Uppsala University, SE-75120 Uppsala, Sweden \\ ${ }^{d}$ Oak Ridge National Laboratory, Oak Ridge TN 54321, USA
}

\begin{abstract}
The aim of this study is to characterize some atomic-scale properties of Ni-based FCC multicomponent alloys. For this purpose, we use Monte Carlo method combined with density functional theory calculations to study short-range order (SRO), atomic displacements, electronic density of states, and magnetic moments in equimolar ternary $\mathrm{NiCrCo}$, and quaternary $\mathrm{NiCrCoFe}$ alloys. According to our study, the salient features for the ternary alloy are a negative SRO parameter between $\mathrm{Ni}-\mathrm{Cr}$ and a positive between $\mathrm{Cr}-\mathrm{Cr}$ pairs as well as a weakly magnetic state. For the quaternary alloy we predict negative SRO parameter for $\mathrm{Ni}-\mathrm{Cr}$ and $\mathrm{Ni}-\mathrm{Fe}$ pairs and positive for $\mathrm{Cr}-\mathrm{Cr}$ and $\mathrm{Fe}-\mathrm{Fe}$ pairs. Atomic displacements for both ternary and quaternary alloys are negligible. In contrast to the ternary, the quaternary alloy shows a complex magnetic structure. The electronic structure of the ternary and quaternary alloys shows differences near the Fermi energy between a random solid solution and the predicted structure with SRO. Despite that, the calculated EXAFS spectra does not show enough contrast to discriminate between random and ordered structures. The predicted SRO has an impact on point-defect energetics, electron-phonon coupling and thermodynamic functions and thus, SRO should not be neglected when study-
\end{abstract}

\footnotetext{
* Corresponding author

Email address: artur.tamm@ut.ee (Artur Tamm)
}

Preprint submitted to Acta Materialia

July 30, 2015

(C) 2015. This manuscript version is made available under the Elsevier user license http://www.elsevier.com/open-access/userlicense/1.0/ 
ing properties of these two alloys.

Keywords: High Entropy Alloy, NiCrCo, NiCrCoFe, Density Functional Theory, Short-range order, Monte Carlo

\section{Introduction}

In the search of better materials needed in many different industries, such as energy production, scientists have started to explore unconventional concepts of material design. Some examples of these are engineered nanostructures and alloys with many different elements. Instead of consisting of two or three principal elements, as is the case with traditional alloys, high entropy alloys (HEA) [1] have five or more elements in close to equimolar ratios that form simple solid solution phases in FCC, HCP, or BCC structures. Recent studies have shown that HEA's have many improved properties compared to conventional alloys or to the pure elements they are made of 2]. For example, high strength, high ductility, better resistance to wear and corrosion.

The core effects that are considered to affect the microstructure and properties of HEA's are [3]: high entropy, sluggish diffusion, severe lattice distortion, and cocktail effect. The high number of principal elements increases the contribution to the free energy from the configurational entropy term, which stabilizes the random solid solution phase with respect to competing phases. The large fluctuations of the lattice potential between different lattice sites hinder the diffusion of atoms by creating a distribution of activation energies. Also, many low potential lattice sites act as traps and further decrease diffusivity. This has been observed in NiCrCoFeMn HEA [4] where the activation energies were higher than in pure elements and ternary alloys consisting of same elements. Due to differences in atomic sizes and bonding the lattice becomes distorted, increasing hardness and strength and making these properties insensitive to

temperature. Lastly, the cocktail effect provides guidelines for manipulating material properties by adding or removing elements, which stabilize BCC or FCC phases, or improve hardness. 
When creating ternary and higher element phase diagrams it is often assumed that the solid solution phases are random. This simplifying assumption is due to the lack of experimental data as is the case for NiCrCo ternary [5] or $\mathrm{NiCrCoFe}$ quaternary alloys. Experimental phase diagram determinations show that the equimolar NiCrCo alloy is a solid solution in FCC phase [6, 7, 8, but no information on the detailed ordering of atoms is available. In fact, short-range order (SRO) has been observed in the binary $\mathrm{NiCr}$ alloy at various compositions and temperatures [9, 10, 11], which suggests that ordering between Ni-Cr pairs might also occur in the ternary and quaternary alloy. This kind of ordering could lower the configurational entropy from its maximum value, which corresponds to a random alloy, and change the expressions for the free energy and the low- $\mathrm{T}$ phase diagram. SRO also decreases the enthalpy of the system, affecting defect energetics.

Computer simulations is a powerful technique to study structure and properties of a material with such an accuracy that for many systems prior experimental knowledge is not needed. It allows screening different structures and studying stability and other properties, without costly experiments. Recently, a hybrid Monte Carlo (MC) and molecular dynamics (MD) method combined with density functional theory calculations (DFT) [12] was used to study refractory HEA [13]. It was shown that the method could be used to study ordering in an alloy at temperatures as low as $300 \mathrm{~K}$.

In this work we explore the short-range order of ternary and quaternary $\mathrm{Ni}$ based high entropy alloys using MC combined with ab initio energetic calculations. We first use lattice MC to find the equilibrium structure of the alloys, and then we use MD to allow for atomic relaxations. With this information we study formation energy, electronic density of states (DOS), magnetic moments and configurational entropy for both random alloy and the structure with the SRO for which no experimental results are available. 


\section{Methodology}

A rule of thumb in $\mathrm{MC}$ calculations suggests that a number as high as $10^{4}$ MC steps per atom have to be attempted in order to approach thermodynamic equilibrium. MC simulations based on $a b$ initio energetics cannot reach such a number of steps, implying that the results are not necessarily converged and must be interpreted with caution. Particularly, we observe clear tendencies for the energetics and SRO, but the values we obtain can not be taken as converged values; longer simulations may results in even larger degrees of order and lower formation energies. Along similar arguments, the combination of MC and MD gives thermodynamic equilibrium structures including relaxations; however, and in order to accelerate convergency, we have verified a posteriori that relaxations account for a small amount of energy, and have therefore conducted lattice MC followed by MD relaxation only at the end of the MC runs.

The systems studied were equimolar $N i_{0.33} \mathrm{Cr}_{0.33} \mathrm{Co}_{0.33}$ ternary, and $\mathrm{Ni}_{0.25} \mathrm{Cr}_{0.25} \mathrm{Co}_{0.25} \mathrm{Fe}_{0.25}$ quaternary alloys in the FCC phase. The temperatures investigated were 500, 800 and 1200K. Energy calculations were conducted using DFT with the VASP software package [14, 15, 16, 17]. The supercell used consisted of 108 atoms. The plane wave cut-off energy in all calculations was $269 \mathrm{eV}$ which is the value recommended for Ni by software manual and is the highest recommended cut-off for the elements under consideration. Methfessel-Paxton smearing method of the first order [18] with a smearing width of $0.1 \mathrm{eV}$ was used, and integration in the Brillouin zone was performed with a mesh created using Monkhort-Pack [19] method with $2 \times 2 \times 2$ points. Core electrons were described with projector augmented-wave pseudopotentials [20, 21] provided by VASP package. The number of valence electrons were 10, 6, 9, and 8 for $\mathrm{Ni}, \mathrm{Cr}$, Co, and Fe respectively. Generalized gradient approximation as described by Perdew-BurkeErnzerhof (PBE) [22] was used for descibing exchange-correlation of electrons. All calculations done were spin-polarized.

Additionally, the calculations to investigate formation energy, structure relaxations, and DOS were conducted with higher number of k-points $(3 \times 3 \times 3)$ to 
increase the accuracy. Convergence studies were made to determine the best calculation parameters needed for accurate results. The energies obtained from the selected parameters have an average error of about $0.2 \mathrm{meV}$ per atom. The X-ray absorption fine structure spectrum for the NiCrCoFe HEA was calculated using ab initio real space multiple scattering calculations as described in [23, 24, 25].

Lattice MC simulations included swap of atomic type trials with the acceptance probability based on the Metropolis - Hastings sampling [26]. In some cases, ionic relaxations at intermediate steps during the simulation were done to assess the change in energy from relaxation. As the atoms are of similar size the resulting changes were of the order of $5 \mathrm{meV}$ per atom, a value that we consider small compared to the gain obtained from chemical ordering, which typically is ten times larger. A special quasi-radom structure (SQS) [27] was selected as an initial starting point in all calculations.

The Warren-Cowley SRO parameter [28] was used to quantify the chemical ordering around an atomic species. The $\operatorname{SRO} \alpha_{i j}^{\nu}$ was calculated using equation 1. where $p_{i j}^{\nu}$ is the probability of finding atomic species $j$ around an atom of type $i$ in the $\nu$ 's neighboring shell and $c_{j}$ is the atomic concentration of type $j$. The SRO $\alpha=0.0$ corresponds to a random solution. Positive values show a tendency to decrease the number of $i, j$ pairs, while negative values correspond to the opposite.

$$
\alpha_{i j}^{\nu}=1-\frac{p_{i j}^{\nu}}{c_{j}}
$$

The SQS structures were created by swapping element species randomly in an initial randomly generated sample until a SRO as close as possible to that of a random alloy up to the third nearest neighbor would be found. In this approach a cost function defined as the sum of SRO squares was minimized to find a structure that would be as close as possible to an ideal random solution for this small sample size.

The configuration entropy of a system with non-zero SRO can be estimated 
using the cluster variation method (CVM) in the pair approximation [29] using the following equation:

$$
S=k_{B}\left((z-1) \sum_{i}\left(c_{i} \ln c_{i}-c_{i}\right)-z / 2 \sum_{i j}\left(y_{i j} \ln y_{i j}-y_{i j}\right)+(z / 2-1)\right),
$$

where $z$ is the number of nearest-neighbors, $c_{i}$ is the concentration of type $i$ and $y_{i j}$ is the probability of finding an $i, j$ pair.

Formation energies were calculated using equation 3 , where $E_{\text {tot }}$ is the total energy per atom of the mixed system, $c_{i}$ is the concentration of element $i$ and $E_{i, r e f}$ is the energy per atom of the reference phase of element $i$. Summation goes over all the elements in the alloy. The reference energies were calculated in the ground state phases of $\mathrm{Ni}, \mathrm{Cr}, \mathrm{Co}$, and Fe, which are $\mathrm{FCC}, \mathrm{BCC}, \mathrm{HCP}$, and $\mathrm{BCC}$ respectively, including magnetism. The lattice constants used for the pure element reference energies are obtained from the equation of state minima predicted by DFT. The calculated magnetic moments of pure element ground states agree well with similar calculations from the literature.

$$
\Delta E_{f}=E_{t o t}-\sum_{i}^{N} c_{i} E_{i, r e f}
$$

\section{Results and discussion}

\subsection{Ternary $\mathrm{NiCrCo}$}

Lattice MC simulations for the NiCrCo equimolar alloy at three temperatures $500 \mathrm{~K}, 800 \mathrm{~K}$, and $1200 \mathrm{~K}$ were performed. The simulations started from a SQS structure using the $3.56 \AA$ experimental lattice parameter [30. The simulation at $500 \mathrm{~K}$ lasted for 1500 steps, which represents 14 swap trials per atom. Higher temperatures were run for $1000 \mathrm{MC}$ steps. The resulting final structure with SRO at 500K and initial SQS structure were later relaxed keeping the volume constant at $0 \mathrm{~K}$ and analyzed.

At 500K the most significant trends in the development of SRO's as well as potential energy are shown on Figure 1 . Although, the number of swap trials per 
Figure 1: Evolution of potential energy and SRO in the lattice MC simulation of $\mathrm{NiCrCo}$ ternary alloy at $500 \mathrm{~K}$. Plotted is the relative potential energy (top) and SRO parameter in the first neihgbor shell for $\mathrm{Cr}-\mathrm{Cr}$ and $\mathrm{Ni}-\mathrm{Cr}$ (bottom) pairs.
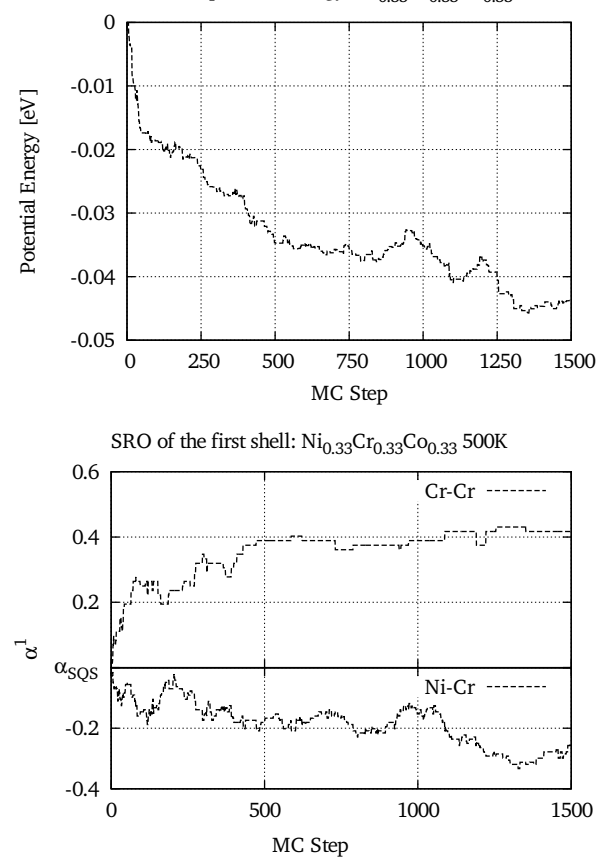
atom is relatively small compared to classical MC simulations the potential energy curve seems to be relatively converged. Our finding is that major deviations from a random solid solution appear for the $\mathrm{Ni}-\mathrm{Cr}$ and $\mathrm{Cr}-\mathrm{Cr}$ pairs. The average number of $\mathrm{Cr}-\mathrm{Cr}$ neighbors is reduced by $\sim 40 \%$, from 4 for the random case to about 2.3, and the number of $\mathrm{Ni}-\mathrm{Cr}$ pairs is increased to 5. Additionally, the number of Ni-Ni pairs is decreased and $\mathrm{Cr}-\mathrm{Co}$ pairs increased slightly. All the other pairs are close to the values of the random solution. With increasing temperature the SRO parameters approach the value of random solid solutions, with the exception of $\mathrm{Cr}-\mathrm{Cr}$ pairs, which still remains significantly high, but somewhat lower than for the $500 \mathrm{~K}$ case. The final averaged SRO parameter values are shown in Table 1 .

The appearance of the SRO reduces the free energy mainly by lowering the formation energy, which changes from 93 to $47 \mathrm{meV}$ per atom. Furthermore, atomic relaxation reduces the energy by no more than $5 \mathrm{meV}$ per atom. Positive formation energy implies a tendency to phase separation at low temperature, but for this small sample size MC is not an adequate technique to explore this phase boundary. The experimental phase diagram at $800^{\circ} \mathrm{C}$ for the ternary $\mathrm{NiCrCo}$ alloy suggests that at the equimolar ratio the system forms an FCC solid solution [5]. We note that the presence of SRO may influence the location of phase boundaries, because of the lower formation energy and configurational entropy.

The large positive value of SRO for $\mathrm{Cr}-\mathrm{Cr}$ pairs might be related to a similar effect observed in Fe-Cr BCC alloy [31]. The change in SRO in that system is related to a strong $\mathrm{Cr}-\mathrm{Cr}$ repulsion when at nearest neighbor positions originated in the magnetic frustration resulting from $\mathrm{Cr}$ atoms forced to align ferromagnetically.

The atomic displacements with respect to perfect lattice positions are found to be in the range of $0.04 \AA$ which are comparable to the magnitude associated to thermal fluctuations and thus are negligible for both the SQS and SRO structures. The largest displacements were mostly related to $\mathrm{Cr}$ atoms. This justifies the use of lattice MC for the ternary system. 
The DOS, for the random alloy and the predicted SRO structure are shown on Figure 2 DOS is not significantly affected by SRO, the largest difference being near the Fermi energy, which has implications for both electric and thermal transport properties, and on the electron-phonon interaction.

The system with SRO has a lower mean magnetic moment than in the case of SQS, as shown in Table 2 The mean magnetic moments of both Ni and Co are lower and mean magnetic moment of $\mathrm{Cr}$ is higher compared to SQS. In the analysis of individual magnetic moments, which are shown in Figure 3 , it can be seen that the main change occurs in the amplitude of the magnetic moments of $\mathrm{Cr}$ atoms. In the case of SQS, the values are closer to a random distribution than in the case of the structure with SRO. Also, magnetic moments of $\mathrm{Cr}$ atoms are not in the same direction, whereas the moments of $\mathrm{Ni}$ and Co are mostly pointing in the same directions.

Finally, we find that the configurational entropy of the alloy with SRO is about $10 \%$ lower than the value of a random solid solution, as reported in Table 2. 


\begin{tabular}{lrrrrrrrrr}
\hline System & Ordering & $E_{f}$ & $S_{\text {conf }}$ & $\Delta r_{r m s}$ & $\langle\mu\rangle$ & $\left\langle\mu_{N i}\right\rangle$ & $\left\langle\mu_{C r}\right\rangle$ & $\left\langle\mu_{C o}\right\rangle$ & $\left.<\mu_{F e}\right\rangle$ \\
& & {$\left[\frac{m e V}{\text { atom }}\right]$} & {$\left[\frac{k_{B}}{\text { atom }}\right]$} & {$[\AA]$} & {$\left[\mu_{B}\right]$} & {$\left[\mu_{B}\right]$} & {$\left[\mu_{B}\right]$} & {$\left[\mu_{B}\right]$} & {$\left[\mu_{B}\right]$} \\
\hline \multirow{2}{*}{$\mathrm{NiCrCo}$} & SQS & 93.0 & 1.099 & 0.044 & 0.332 & 0.193 & -0.229 & 1.031 & \\
& $\mathrm{SRO}$ & 47.3 & 0.944 & 0.035 & 0.080 & 0.098 & -0.857 & 0.999 & \\
\multirow{2}{*}{$\mathrm{NiCrCoFe}$} & $\mathrm{SQS}$ & 76.7 & 1.386 & 0.057 & 0.668 & 0.319 & -0.903 & 1.173 & 2.083 \\
& $\mathrm{SRO}$ & 15.8 & 1.097 & 0.031 & 0.537 & 0.296 & -1.461 & 0.983 & 2.330 \\
\hline
\end{tabular}

Table 2: Properties of NiCrCo ternary and $\mathrm{NiCrCoFe}$ quaternary systems for the special quasirandom structure (SQS) and structure with ordering (SRO) as predicted by lattice MC simulations at $500 \mathrm{~K}$. The values are obtained for $0 \mathrm{~K}$ relaxed structures at constant volume.

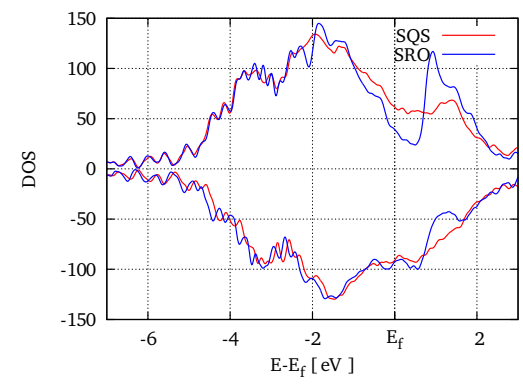

Figure 2: Electronic density of states for the initial quasi-random structure (SQS) and the structure predicted by lattice MC simulation (SRO) of $\mathrm{NiCrCo}$ ternary system relaxed at $0 \mathrm{~K}$. 
Figure 3: Magnetic moments of individual atoms in quasi-random structure (SQS) and structure with short-range order (SRO) as predicted by lattice MC at $500 \mathrm{~K}$ for the equimolar $\mathrm{NiCrCo}$ alloy.

\subsection{Quaternary $\mathrm{NiCrCoFe}$}

Similar lattice MC simulations were done with the quaternary $\mathrm{NiCrCoFe}$ alloy. The simulations started from SQS structure with an experimental lattice parameter of $3.57 \AA$ [30] and the $500 \mathrm{~K}$ case ran for 2000 steps resulting in 18 swap trials per atom. The higher temperature runs lasted for 1500 steps. The final SRO parameters at three temperatures are provided in Table 3 . Also, the evolution of potential energy and major trends in the evolution of SRO are plotted in Figure 4. Here again the evolution of potential energy suggests that the simulation has been long enough to show a clear trend. The final structure from the $500 \mathrm{~K}$ simulations was relaxed at $0 \mathrm{~K}$ without changing the volume and analyzed.

Similar trends as in the case of ternary alloy are clearly present. The number of $\mathrm{Cr}-\mathrm{Cr}$ pairs is reduced by $\sim 50 \%$, and the number of $\mathrm{Ni}-\mathrm{Cr}$ is increased, when compared to a random alloy. Additionally, the number of Fe-Fe pairs is significantly reduced and number of $\mathrm{Ni}-\mathrm{Fe}, \mathrm{Cr}-\mathrm{Co}$, and $\mathrm{Co}-\mathrm{Fe}$ pairs is increased. Also, there is quite a large reduction of $\mathrm{Ni}-\mathrm{Ni}$ and $\mathrm{Co}-\mathrm{Co}$ pairs, where the number of same type neighbors is reduced to 2 atoms instead of 3 . Similarly to the case of the ternary alloy, the increase in temperature reduces the SRO values of all pairs, while the value for the $\mathrm{Cr}-\mathrm{Cr}$ pairs is still significantly large. 
Figure 4: Development of potential energy and SRO in the lattice MC simulation of $\mathrm{NiCrCoFe}$ quaternary alloy at $500 \mathrm{~K}$. Plotted is the relative potential energy (top) and SRO parameter in the first neihgbor shell for $\mathrm{Cr}-\mathrm{Cr}$ and $\mathrm{Fe}-\mathrm{Fe}$ pairs (bottom).
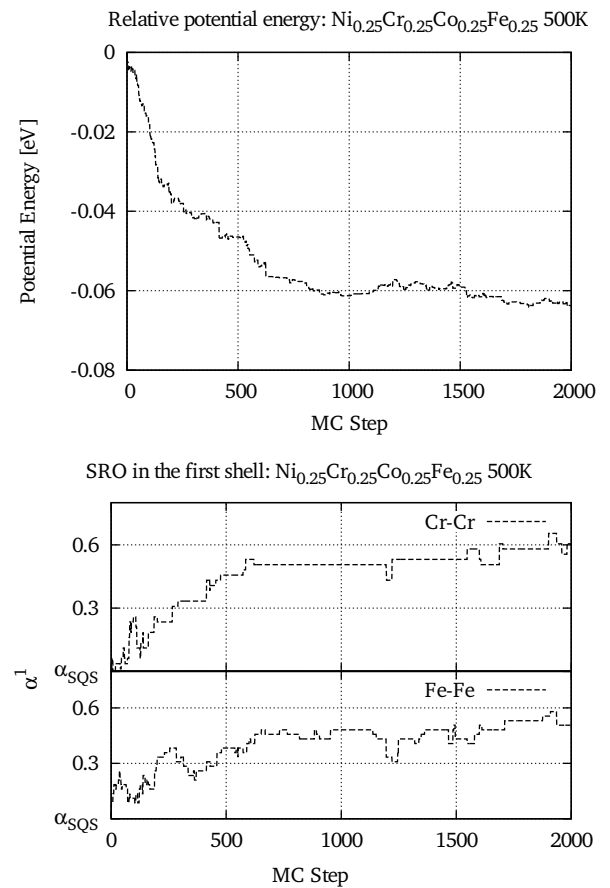
The deviation of SRO for Cr-Cr pairs is even larger for the quaternary case than for the ternary. Investigation of SRO for second and third shells in the $1200 \mathrm{~K}$ case revealed that most of the Cr atoms are at the second nearest neighbor locations, implying the formation of an ordered structure with simple cubic Cr sublattice. As for the ternary alloy, the magnetic frustration of $\mathrm{Cr}$ atoms leads to structure where every $\mathrm{Cr}$ atom is surrounded by ferromagnetic elements. The inclusion of Fe into alloy enhances this kind of driving force even more.

As in the case of ternary alloy, the development of SRO involves a lowering of formation energy by about $60 \mathrm{meV}$ per atom, from 76 to 16 . Atomic relaxation changes the energy from its initial value about $5 \mathrm{meV}$ per atom. Although there is a large reduction in formation energy for the final structure it still remains positive and thus, phase separation should occur at low temperatures.

Furthermore, the DOS at the Fermi level differs significantly from the random alloy, as seen in Figure 5. The lower density of states near the Fermi energy would decrease the electron-phonon coupling as it is proportional to DOS.

The magnetic moments of the structure with SRO have a much smaller spread and, as in the ternary alloy, those of $\mathrm{Cr}$ atoms are mostly antiferromagnetically aligned with respect to all other elements. SRO also increases the absolute value of mean magnetic moments.

Similarly with the ternary alloy, SRO results in a sizable change of the configurational entropy in relation to the random solution, of about $26 \%$ as calculated from the CVM model. This would significantly change the locations of phase boundaries.

Next, during the atomic relaxation at 0K the atoms move only slightly and the mean atomic displacement is around $0.06 \AA$. As with the ternary alloy the largest displacements are for $\mathrm{Cr}$ atoms.

Finally, the EXAFS spectra was calculated for the quaternary case for both SQS and SRO structures. The results are shown in Figure 7 These results agree quite well with experimental results, but there is no significant difference between the SQS and SRO spectra, which suggests that X-ray methods do not show enough contrast between these elements to detect the changes in SRO that 


\begin{tabular}{lccc}
\hline Pair & $500 \mathrm{~K}$ & $800 \mathrm{~K}$ & $1200 \mathrm{~K}$ \\
\hline $\mathrm{Ni}-\mathrm{Ni}$ & 0.30 & 0.16 & 0.13 \\
$\mathrm{Ni}-\mathrm{Cr}$ & -0.18 & -0.21 & -0.22 \\
$\mathrm{Ni}-\mathrm{Co}$ & 0.14 & 0.10 & 0.12 \\
$\mathrm{Ni}-\mathrm{Fe}$ & -0.27 & -0.04 & -0.04 \\
$\mathrm{Cr}-\mathrm{Cr}$ & 0.59 & 0.54 & 0.65 \\
$\mathrm{Cr}-\mathrm{Co}$ & -0.31 & -0.18 & -0.26 \\
$\mathrm{Cr}-\mathrm{Fe}$ & -0.10 & -0.14 & -0.17 \\
Co-Co & 0.33 & 0.13 & 0.11 \\
Co-Fe & -0.16 & -0.05 & 0.03 \\
Fe-Fe & 0.54 & 0.23 & 0.17 \\
\hline
\end{tabular}

Table 3: Short-range order parameter of the NiCrCoFe quaternary FCC system averaged over last steps of the lattice MC simulation at three temperatures.

we predict from lattice MC simulations.

\section{Conclusions}

In summary, lattice $\mathrm{MC}$ simulations were conducted to study $\mathrm{SRO}$ in $\mathrm{Ni}$ based ternary and quaternary alloys, as a preliminary study aiming at Ni-based HEA's. Our main conclusion is that, contrary to the common assumption that HEA's are random solid solutions, equimolar NiCrCo HEA alloy shows a significant degree of short range order involving a $\sim 40 \%$ decrease in the number of $\mathrm{Cr}-\mathrm{Cr}$ pairs together with an increase in $\mathrm{Ni}-\mathrm{Cr}$ and $\mathrm{Cr}$-Co pairs with respect to random distributions. For the $\mathrm{NiCrCoFe}$ quaternary alloy a similar behavior was found, namely an even larger decrease in the number of $\mathrm{Cr}-\mathrm{Cr}$ and $\mathrm{Fe}-\mathrm{Fe}$ pairs and a moderate increase in $\mathrm{Ni}-\mathrm{Cr}, \mathrm{Ni}-\mathrm{Fe}, \mathrm{Cr}-\mathrm{Co}$, and $\mathrm{Co}-\mathrm{Fe}$ pairs as well 
Figure 5: Electronic density of states for the initial quasi-random structure (SQS) and the structure predicted by lattice $\mathrm{MC}$ simulation (SRO) of $\mathrm{NiCrCoFe}$ quaternary system relaxed at $0 \mathrm{~K}$.

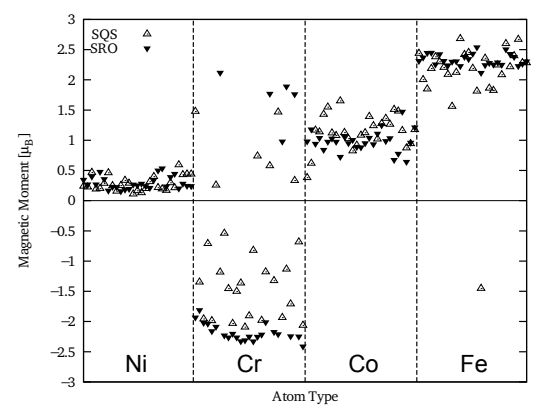

Figure 6: Magnetic moments of individual atoms in quasi-random structure (SQS) and structure with short-range order (SRO) as predicted by lattice MC at $500 \mathrm{~K}$ for the equimolar $\mathrm{NiCrCoFe}$ alloy. 
Figure 7: Calculated EXAFS of quasi-random (SQS) and structure predicted by lattice $\mathrm{MC}$ simulation (SRO) for the equimolar $\mathrm{NiCrCoFe}$ alloy in comparison with experimental data.

as a decrease of Ni-Ni and Co-Co pairs. We also show that this SRO affects properties such as alloy formation energy, atomic mean square displacements, electronic density of states, and configurational entropy. Ordering lowers the formation energy in several tenths of meV per atom and reduces the configurational entropy by $\sim 20 \%$, enough to change the values of the free energy of the solution phase affecting the location of phase boundaries by significant amounts. Additionally, SRO defines the energy landscape for defects in both their formation and migration energies. Lastly, the change in DOS near the Fermi energy due to SRO influences transport properties and the electro-phonon coupling.

\section{Acknowledgements}

This work was supported by the Energy Deposition to Defect Evolution (EDDE), an Energy Frontier Research Center funded by the U.S. Department of Energy, Office of Science, Basic Energy Sciences (Award Number 2014 ORNL 1026) at Oak Ridge and Los Alamos National Laboratories. AT was partially funded by by the European Social Fund's Doctoral Studies and Internationalization Programme DoRa, which is carried out by Foundation Archimedes and Estonian Research Council grant IUT20-24. 
[1] J. W. Yeh, Y. L. Chen, S. J. Lin, S. K. Chen, High-Entropy Alloys - A New Era of Exploitation, Mater. Sci. Forum 560 (2007) 1-9. doi:10.4028/www. scientific.net/MSF.560.1

[2] Y. Zhang, T. T. Zuo, Z. Tang, M. C. Gao, K. a. Dahmen, P. K. Liaw, Z. P. Lu, Microstructures and properties of high-entropy alloys, Prog. Mater. Sci. 61 (October 2013) (2014) 1-93. doi:10.1016/j.pmatsci.2013.10.001

[3] J.-W. Yeh, Alloy Design Strategies and Future Trends in High-Entropy Alloys, Jom 65 (12) (2013) 1759-1771. doi:10.1007/s11837-013-0761-6

[4] K.-Y. Tsai, M.-H. Tsai, J.-W. Yeh, Sluggish diffusion in Co-Cr-Fe-Mn-Ni high-entropy alloys, Acta Mater. 61 (13) (2013) 4887-4897. doi:10.1016/ j.actamat.2013.04.058.

[5] S. Yang, M. Jiang, H. Li, Y. Liu, L. Wang, Assessment of Co-Cr-Ni ternary system by CALPHAD technique, Rare Met. 31 (1) (2012) 75-80. doi: $10.1007 / \mathrm{s} 12598-012-0466-\mathrm{y}$

[6] S. Rideout, W. D. Manly, E. L. Kamen, B. S. Lement, P. A. Beck, Intermediate Phases in Ternary Alloy Systems of Transition Elements, Trans AIME 191 (1951) 872-876.

[7] G. P. Zhmurko, E. G. Kabanova, V. N. Kuznetsov, a. V. Leonov, Phase equilibria in the Co-Cr-Ni system, Moscow Univ. Chem. Bull. 63 (4) (2008) 234-235. doi:10.3103/S0027131408040135.

[8] T. Omori, J. Sato, K. Shinagawa, I. Ohnuma, K. Oikawa, R. Kainuma, K. Ishida, Experimental determination of phase equilibria in the Co-Cr-Ni system, J. Phase Equilibria Diffus. 35 (2) (2014) 178-185. doi:10.1007/ s11669-014-0292-z.

[9] W. Schweika, H. Haubold, Neutron-scattering and Monte Carlo study of short-range order and atomic interaction in Ni 0.89 Cr 0.11, Phys. Rev. B 37 (16) (1988) 9240-9248. 
[10] B. Schönfeld, L. Reinhard, Short-Range Order and Atomic Displacements in Ni-20 at\% Cr Single Crystals, Phys. status solidi 148 (1988) 457-471.

[11] R. Caudron, M. Sarfati, In situ diffuse scattering of neutrons in alloys and application to phase diagram determination, J. Phys. I Fr. 2.

[12] W. Kohn, L. J. Sham, Self-Consistent Equations Including Exchange and Correlation Effects, Phys. Rev. 140 (1965) A1133. doi:10.1103/PhysRev. $140 . \mathrm{A} 1133$.

[13] M. Widom, W. P. Huhn, S. Maiti, W. Steurer, Hybrid Monte Carlo/Molecular Dynamics Simulation of a Refractory Metal High Entropy Alloy, Metall. Mater. Trans. A 45 (1) (2013) 196-200. doi:10.1007/ s11661-013-2000-8.

[14] G. Kresse, J. Hafner, Ab initio molecular dynamics for liquid metals, Phys. Rev. B 47 (1).

[15] G. Kresse, J. Hafner, Ab initio molecular-dynamics simulation of the liquidmetal-amorphous-semiconductor transition in germanium, Phys. Rev. B 49 (1994) 14251.

[16] G. Kresse, J. Furthmuller, Efficiency of ab-initio total energy calculations for metals and semiconductors using a plane-wave basis set, Comp. Mater. Sci. 6 (1996) 15.

[17] G. Kresse, J. Furthmüller, Efficient iterative schemes for ab initio totalenergy calculations using a plane-wave basis set., Phys. Rev. B. Condens. Matter 54 (16) (1996) 11169-11186.

[18] M. Methfessel, A. T. Paxton, High-precision sampling for Brillouin-zone integration in metals, Phys. Rev. B 40 (6) (1989) 3616-3621.

[19] H. Monkhorst, J. Pack, Special points for Brillouin-zone integrations, Phys. Rev. B 13 (1976) 5188-5192. 
[20] P. E. Blöchl, Projector augmented-wave method, Phys. Rev. B 50 (1994) 17953. doi:10.1103/PhysRevB.50.17953.

[21] G. Kresse, D. Joubert, From ultrasoft pseudopotentials to the projector augmented-wave method, Phys. Rev. B 59 (1999) 1758.

[22] J. P. J. Perdew, K. Burke, M. Ernzerhof, Generalized Gradient Approximation Made Simple, Phys. Rev. Lett. 77 (18) (1996) 3865.

[23] A. L. Ankudinov, B. Ravel, J. J. Rehr, S. D. Conradson, Real-space multiple-scattering calculation and interpretation of x-ray-absorption nearedge structure, Phys. Rev. B 58 (1998) 7565-7576.

[24] J. J. Rehr, R. C. Albers, Theoretical Approaches to X-ray Absorption Fine Structure, Rev. Mod. Phys. 72 (2000) 621.

[25] J. J. Rehr, J. J. Kas, M. P. Prange, A. P. Sorini, Y. Takimoto, F. D. Vila, Ab initio theory and calculations of X-ray spectra, Comptes Rendus Physique 10 (6) (2009) 548-559.

[26] W. K. Hasting, Monte Carlo sampling methods using Markov chains and their applications, Biometrica 57 (1970) 97.

[27] A. Zunger, S. H. Wei, L. G. Ferreira, J. E. Bernard, Special quasirandom structures, Phys. Rev. Lett. 65 (3) (1990) 353-356.

[28] J. M. Cowley, An approximate theory of order in alloys, Phys. Rev. 77 (5) (1950) 669-675. doi:10.1103/PhysRev.138.A1384

[29] R. Kikuchi, CVM Entropy Algebra, Prog. Theor. Phys. Suppl. 115 (1994) $1-26$.

[30] M. Stocks, private communication.

[31] A. Caro, M. Caro, P. Klaver, B. Sadigh, The computational modeling of alloys at the atomic scale: From ab initio and thermodynamics to radiationinduced heterogeneous precipitation, J. Miner. Met. Mater. Soc. 59 (April) (2007) 52. doi:10.1007/s11837-007-0055-y 


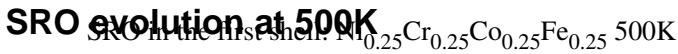

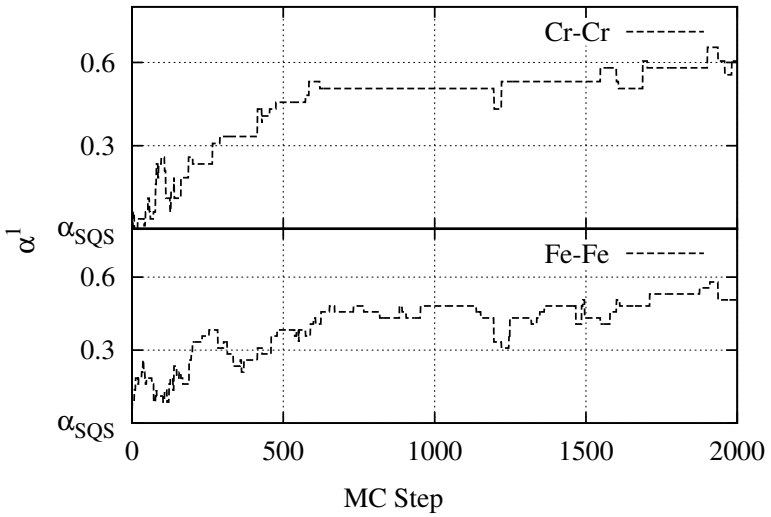

